\title{
Clinical Spectrum and Neuroimaging Changes in Neurodegenerative Disorder-Experiences in Tertiary Care Hospital of Bangladesh
}

\author{
Gopen Kumar Kundu, Rumana Islam, Noor- E-Sabah, ABM Mukib
}

\section{ABSTRACT}

\begin{abstract}
Neurodegenerative diseases (NDD) are a heterogeneous group of disorders characterized by progressive loss of previously acquired skills that are of varied etiology, clinical manifestations, and natural course. There is a paucity of data on clinical profile of neurodegenerative diseases in our population.
\end{abstract}

We conducted a retrospective study with 68 diagnosed cases of NDD at a tertiary care hospital in Bangladesh. Among them, more than one-third of children were in 1-5 years age group. The mean age was 10.2 \pm 3.1 year and male to female ratio was $2: 1$. Fifty percent of cases had a history of consanguineous parents. Leukodystrophy was most common $(30.88 \%)$ among NDDs, followed by Wilson disease (26.47), SSPE (22.1\%), and Degenerative Ataxia (20.59\%).

Motor skill regression was the most common presentation (97\%), followed by speech regression in $91 \%$ and Gait disorder in $83 \%$ of children. Seizure was presenting features in $24 \%$ of children. Neuroimaging abnormalities were found in $\mathbf{8 0 . 8 8 \%}$ NDD cases. Among them white matter hyper intensity in $29.41 \%$, cerabeller atrophy in $13.25 \%$, and cerebral atropy in $11.76 \%$ of children. Eye changes were found in about two-thirds $(69.12 \%)$ of cases of NDD. Among them, optic atrophy was found in $29.41 \%$, and $\mathrm{KF}$ rings in $25.00 \%$ of cases.

Keywords: Neurodegenerative disease, neuroimaging, ophthalmological findings.
Published Online: November 23, 2021

ISSN: $2736-5476$

DOI: 10.24018 /ejclinicmed.2021.2.6.143

\section{G. Kumar Kundu*}

Department of Pediatric Neurology, Bangabandhu Sheikh Mujib Medical University, Dhaka, Bangladesh.

(e-mail: gopen.kundu@gmail.com)

R. Islam

Department of Pediatric Neurology, Bangabandhu Sheikh Mujib Medical University, Dhaka, Bangladesh.

N. E-Sabah

Department of Pediatric Neurology, Bangabandhu Sheikh Mujib Medical University, Dhaka, Bangladesh.

A. Mukib

Department of Pediatric Neurology, Bangabandhu Sheikh Mujib Medical University, Dhaka, Bangladesh.

*Corresponding Author

\section{INTRODUCTION}

Neurodegenerative diseases (NDDs) are not an uncommon clinical entity encountered in Pediatric neurology practice. However, it is an umbrella term that consists of a heterogeneous group of disease entities. In the UK-wide study of progressive intellectual and neurological deterioration (PIND) in children between 1997 and 2014 showed that the leukodystrophies and genetic leukoencephalopathies constitute a significant proportion of pediatric neurodegenerative disease in the UK [1]. The relative percentage of illnesses that make up the burden of NDD in any given population is useful in developing diagnostic and therapeutic services. NDDs in children may have combined maldevelopment and degenerative features or often multisystem involvement [2].

The hallmark of a neurodegenerative disease is regression and progressive deterioration of neurologic function with loss of speech, vision, hearing, or locomotion, often associated with seizures, feeding difficulties, and impairment of intellect3. They can be divided into predominantly grey matter or white matter involved NDDs and associated with systemic manifestations. The relative distribution of the various causes of NDDs is different in different populations.
Epidemiologic data about the commonly prevalent disease entities in the local population helps to guide diagnostic tests so that limited resources are used efficiently. There is a paucity of data on clinical profile of neurodegenerative diseases among the Bangladeshi population. Therefore, the present study was planned to obtain data on the clinical, biochemical, and neuroradiological profile of NDDs in the patients presenting in a tertiary care hospital of Bangladesh.

\section{Methodology}

This was a hospital based retrospective study. The case files of all NDDs cases were registered in the Department of Pediatric Neurology of BSMMU hospital over five years (2016-2020). Then these data were retrieved and analyzed after institutional ethical clearance. Any child (under 16 years of age at onset of symptoms) who fulfills the following criteria: progressive deterioration for more than 3 months with loss of already attained intellectual or developmental abilities and development of abnormal neurological signs were included in the study. The pre-symptomatic individuals identified with diagnosed neurodegenerative conditions were also included in the study.

Children with static (non-progressive) intellectual / 
neurological loss are excluded. Clinical information is obtained via a questionnaire completed by the notifying pediatrician. Clinical presentations, eye findings, biochemical and neuroimaging profiles were further assessed.

\section{RESULTS}

The total number of patients was 68 . Among them, more than one-third of children $(36.75 \%)$ were in $1-5$ years age group. Male patients (57.35\%) were outnumbered than female $(42.64 \%)$. Male-female ratio was $1.3: 1$. About twothirds $(61.76 \%)$ of patients belonged to lower middle-class group. About two-thirds of patients $(60.29 \%)$ were from rural area and $39.71 \%$ were from urban. Fifty percent (50\%) cases had a history of consanguineous parents. A majority $(92.64 \%)$ of cases had uneventful perinatal history.

Among total NDD cases, Leukodystrophy was most common, it was about $30.88 \%$ followed by Wilson disease $26.47 \%$, SSPE 22.7\%, and Degenerative Ataxia 20.59\%. Regarding presentations of NDD cases, Motor skill regression was found in $97 \%$ of children, Speech regression in $91 \%$ of children, Gait disorders in $83 \%$ of children, Ataxia in $20 \%$ of children, Skin pigmentation in $9 \%$ of children, Oculomotor apraxia in 10\% children, Myoclonic jerks in 22 $\%$ children, Seizure in $24 \%$ children and Musculo-skeletal abnormalities (Pes cavas) were found in 4\% NDD children. Among laboratory findings, Anti measles antibody was found in $22.7 \%$ cases, high $\alpha$-feto protein in $11.76 \%$, Abnormally low $\lg \mathrm{A}, \operatorname{lgG}$ in $11.76 \%$ cases, Low serum ceruloplasmin in $26.47 \%$, High ACTH in $4.41 \%$ cases.

Neuroimaging changes were found in $80.88 \%$ NDD cases. Among them white matter hyperintensity in $29.41 \%$, Basal ganglia hyperintensity in $23.53 \%$, Cerebral atrophy in $11.76 \%$, Cerabeller atrophy in $13.25 \%$ cases. Eye changes were found in about two-thirds (69.12\%) of cases of NDD. Among them, KF rings were found in $25.00 \%$ of cases, Optic atrophy in $29.41 \%$, Telangiectasia in $11.76 \%$ of cases.

\begin{tabular}{lcc}
\multicolumn{3}{c}{ TABLE I: BASELINE CHARACTERISTICS OF } \\
STUDIED NDD PATIENTS (N-68)
\end{tabular}

Table I showed more than one-third of children (36.75\%) were 1-5 years age group. The mean age was $10.2 \pm 3.1$. A male patient $(57.35 \%)$ was outnumbered than female (42.64\%). Male-female ratio was 1.3:1. About two-thirds of patients $(61.76 \%)$ patient belonged to lower middle-class group. About two-third of patients $(60.29 \%)$ were from rural area and $39.71 \%$ from urban. Fifty percent (50\%) cases had a history of consanguineous parents. Majority (92.64\%) of cases had uneventful perinatal history.

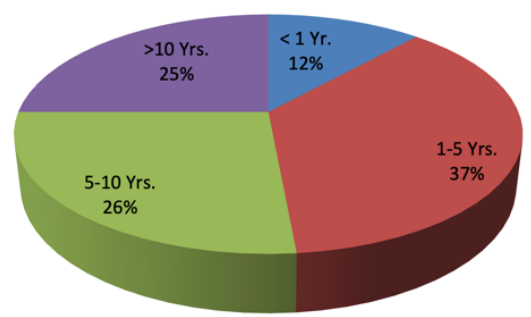

Fig. 1. Pie chart showing age distribution among the study subjects.

TABLE II: TYPE OF STUDIED NEURODEGENERATIVE DISORDERS CASES (N-68)

\begin{tabular}{lcc}
\hline \hline A. Genetic disorders (Leukodystrophy) & 21 & 30.88 \\
Metachromatic leukodystrophy & 11 & 16.17 \\
Adrenoleukodystrophy & 3 & 4.41 \\
Canavan diseases & 3 & 4.41 \\
Vender napp diseases & 1 & 1.47 \\
Krabbe disease & 2 & 2.94 \\
$\quad$ Aicardi Goutiers syndrome & 1 & 1.47 \\
B. Infections disease & & \\
Sub-Acute Sclerosing Panencephalities & 15 & 22.7 \\
C. Inherited Metabolic disorder: & & \\
$\quad$ Wilson disease & 18 & 26.47 \\
D. Degenerative Ataxia: & 14 & 20.59 \\
$\quad$ Ataxia telengiectasis $\quad$ central & 3 & 11.76 \\
Friedrichs ataxia & 2 & 4.41 \\
Childhood ataxia with & & 2.94 \\
hypomyelination (CACH) syndrome & 1 & 1.47 \\
$\quad$ Neuronal CeroidLipofucinosis (NCL)
\end{tabular}

Table II showed the type of studied NDD cases. Leukodystrophy was most common. It was about $30.88 \%$, Wilson disease was $26.47 \%$, SSPE was $22.7 \%$ and Degenerative Ataxia was $20.59 \%$.

Table III showed clinical presentations of NDD patients. Here Motor skill regression was found in $97 \%$ of children, Gait disorder in $83 \%$ children, Speech regression in $91 \%$ children, Ataxia in $20 \%$ children, Skin pigmentation in $9 \%$ children, Oculomotor apraxia in $10 \%$ of children, Myoclonic jerks in $22 \%$ children, Seizure in $24 \%$ children and Musculoskeletal abnormalities (Pes cavas) were found in $4 \%$ NDD children.

TABLE III: CliniCAL PRESENTATIONS OF STUDIED NDD PATIENTS (N-68)

\begin{tabular}{ccc} 
Presentations & Number & $\begin{array}{c}\text { Percentage } \\
(\%)\end{array}$ \\
\hline \hline Motor skill regression & 66 & 97 \\
Gait disorder & 57 & 83 \\
Speech regression & 62 & 91 \\
Ataxia & 14 & 20 \\
Skin pigmentation & 6 & 9 \\
Oculomotor apraxia & 7 & 10 \\
Myoclonic jerks & 15 & 22 \\
Seizure & 16 & 24 \\
Musculoskeletal abnormalities (PesCavas) & 3 & 4 \\
\hline \hline
\end{tabular}


TABLE IV: LABORATORY INVESTIGATIONS OF STUDIED NDD PATIENTS

\begin{tabular}{ccc}
\multicolumn{3}{c}{$(\mathrm{N}-68)$} \\
\hline \hline Lab Investigations & Number & Percentage (\%) \\
\hline \hline Antimeasles antibody lgG +Ve in & 15 & 22.7 \\
CSF & 8 & 11.76 \\
a-feto protein high & 8 & 11.76 \\
Abnormal low lgG\&lgA & 18 & 26.47 \\
Serum Ceretoplasmin low & 3 & 4.41 \\
High ACTH & 5 & 7.35 \\
Low RBS & 7 & 10.3 \\
Reduced NCV & 2 & 2.94 \\
Genetic analysis (ARSA gene \& & 2 & \\
RNASE H2A gene) & & \\
\hline \hline
\end{tabular}

TABLE V: NEUROIMAGING FINDINGS OF STUDIED NDD PATIENTS (N-68)

\begin{tabular}{ccc}
\hline \hline Findings & $\begin{array}{c}\text { Number } \\
\text { Phite matter hyper intensity } \\
(\%)\end{array}$ \\
\hline \hline $\begin{array}{c}\text { (Leukodystrophy, Periventricular } \\
\text { hyperintensity, Central white matter } \\
\text { hyperintencity \& Posterior white matter }\end{array}$ & 20 & 29.41 \\
hyper intensity) & & \\
Cerebral atrophy & 8 & 11.76 \\
Cerabeller atrophy & 9 & 13.25 \\
Basal ganglia hyperintensity & 16 & 23.53 \\
Complete white matter hyper intensity & 2 & 2.94 \\
with CSF density (VWMS) & 13 & $19.12 \%$ \\
Normal & & \\
\hline \hline
\end{tabular}

Table IV showed laboratory investigations of Studied NDD Patients. Anti-measles antibody was found in $22.7 \%$ cases, high $\alpha$-feto protein in $11.76 \%$, Abnormally low $\operatorname{lgA}$, $\operatorname{lgG}$ in $11.76 \%$ cases, Low serum ceruloplasmin in $26.47 \%$, High ACTH in $4.41 \%$ cases, Low RBS in $7.35 \%$ cases, Reduced NCV in $10.3 \%$ cases and Abnormal Genetic mutation was found in $2.94 \%$ cases of NND.

Table $\mathrm{V}$ showed neuroimaging changes in the patients. Neuroimaging changes found in $80.88 \%$ Neurodegenerative disorder of studied patients. Among them, white matter hyperintensity in $29.41 \%$, Basal ganglia hyperintensity in $23.53 \%$ Cerebral atrophy in $11.76 \%$, Cerabeller atrophy in $13.25 \%$, Complete white matter hyperintensity with CSF density (VWMS) found in $2.94 \%$ cases of NDD, and Normal neuroimaging findings were found in $19.12 \%$ patient.

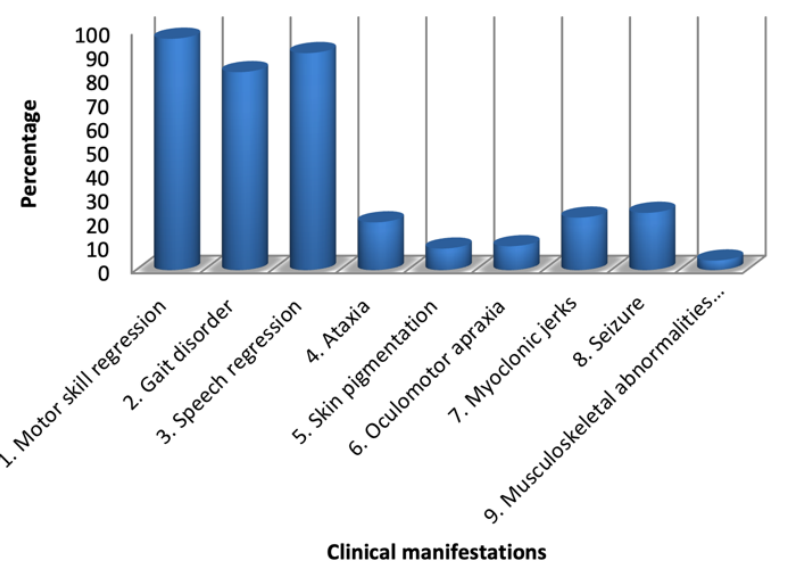

Fig. 2. Column showing the distribution of clinical presentations of NDD patients.

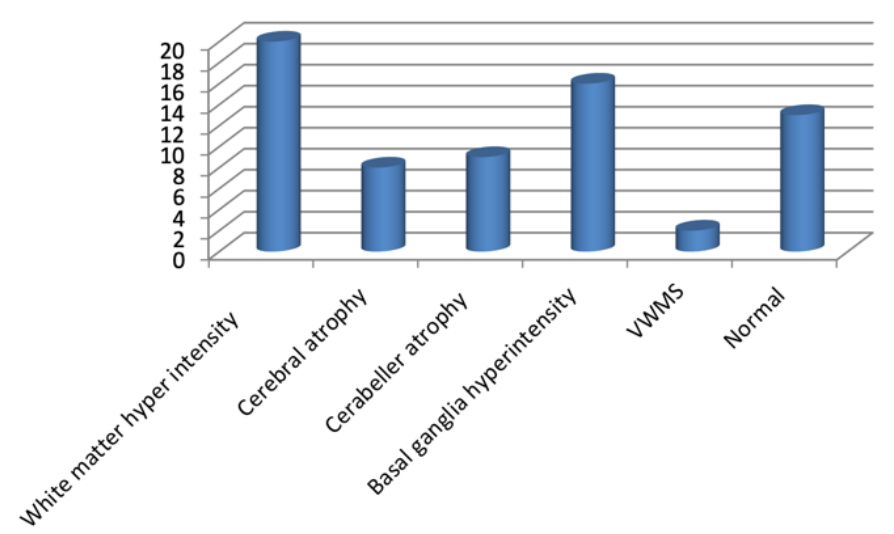

Fig. 3. Column showing the distribution of Neuroimaging findings in patients with NDD.

TABLE VI: OPHTHALMOLOGIC FINDINGS OF STUDIED NDD PATIENTS (N-68)

\begin{tabular}{ccc}
\multicolumn{3}{c}{ STUDIED NDD PATIENTS $(\mathrm{N}-68)$} \\
\hline \hline Findings & Number & Percentage (\%) \\
\hline K F ring & 17 & 25.00 \\
Optic atrophy & 20 & 29.41 \\
Papilloedema & 1 & 1.47 \\
Telengiectasia & 8 & 11.76 \\
Sunflower cataract & 1 & 1.47 \\
Normal & 21 & 30.88 \\
\hline \hline
\end{tabular}

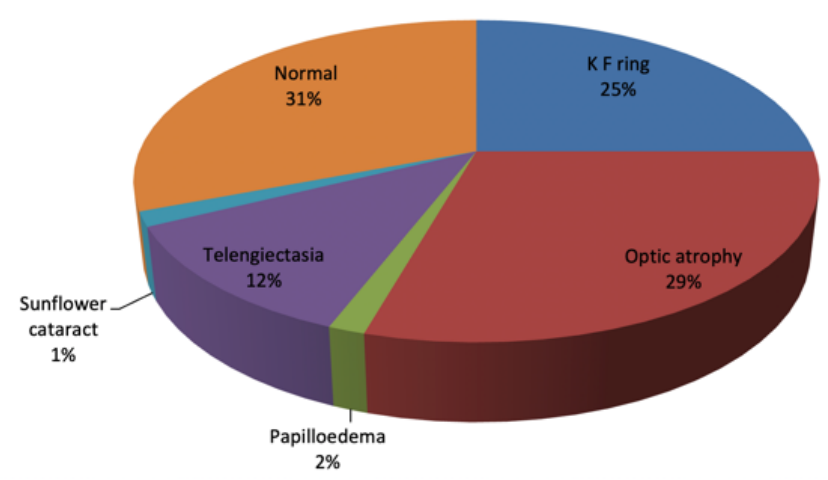

Fig. 4. Pie chart showing the distribution of ophthalmologic findings in studied NDD patients.

Table IV, eye changes found about $69.12 \%$ of patients with NDD. Among them, KF rings were found in $25.00 \%$, Optic atrophy in $29.41 \%$, Telengiectasia in $11.76 \%$, Sunflower cataract in $1.47 \%$, Papilloedema in $1.47 \%$ cases of NDD, and Normal eye evaluation found in $30.88 \%$ cases.

\section{DISCUSSION}

Neurodegenerative diseases (NDDs) are a common clinical entity encountered in pediatric neurology practice. These are a heterogeneous group of diseases marked by progressive loss of milestones but are of diverse etiology, clinical presentation, and natural history. In this study, 68 patients were studied to see the clinical spectrum and neuroimaging changes in neurodegenerative disorder-in Bangabandhu Sheikh Mujib Medical University. In this study, more than one-third of children $(36.75 \%)$ were $1-5$ years age group, male patient $(57.35 \%)$ was outnumbered than female $(42.64 \%)$. Male-female ratio was $1.3: 1$. As from previous knowledge, many cases of leukodystrophy including MLD, ALD, Canavan disease, krabbe disease, Hypomyelinating disorders, Neurodegenaration with brain iron accumulation, 
Lysosomal storage disease, peroxisomal and mitochondrial disorders, glucose metabolism disorders may present within 1-5 years of age [3]. Most of the NDDs in children are inherited with autosomal recessive patterns and in this study, fifty percent $(50 \%)$ cases had history of consanguineous parents. In this study, NDDs are classified into leukodystrophies, infectious disorders, inherited metabolic disorders, and degenerative ataxias. Among them, leukodystrophies were found most prevalent (30.88\%) followed by, inherited metabolic disorders (26.47), infectious disorders (22.7\%), and degenerative ataxias (20.59\%).

In PIND study from 1997 to 2014, 51\% of the diagnosed children had a leukodystrophy or a genetic leukoencephalopathy [1], [4]. Nine commonest leukodystrophies were: metachromatic leukodystrophy (22\%), X-linkedadrenoleukodystrophy (including asymptomatic X-ALD) (21\%), Krabbe disease (16\%), Aicardi-Goutieres disease (7\%), Alexander disease $(6 \%)$, Canavan disease $(5 \%)$, Vanishing whitematter disease $(5 \%)$, Pelizaeus-Merzbacher disease (5\%), and Megalencephalic leukoencephalopathy with subcortical cysts (3\%) [1].

In one retrospective study from North India from January 2008 to December 2012 total of 1,314 children were seen. Out of these, 80 cases were diagnosed as leukodystrophies including MLD, ALD, Alexander disease, Krabbe disease, Canavan disease, vanishing white matter disease, megalencephalic leukoencephalopathy with subcortical cyst, and hypomyelinating disorder [5].

Reference [4], they reviewed the charts of a total of 1218 patients admitted during the ten years, including 548 from Indiana University and 670 from the Medical College of Georgia. Neurodegenerative diseases represented 341(28\%) of the total diagnosed cases including Leukodystrophies, SSPE, Wilson disease, Degenerative ataxias, and other neurodegenerative conditions [6].

Regarding clinical manifestations most patients presented with motor skill regression (97\%), Speech regression (91\%), Gait disorder (83\%), and Ataxia (20\%). Seizure was present in $24 \%$ of children which is an early manifestation of gray matter disease and may be the late presentation of white matter disease. Two patients with VWMD presented with progressive neuroregression following a febrile illness, optic atrophy, relatively preserved cognitive functions and typical MRI features of VWMD. A rare case of Aicardi Goutiers syndrome was diagnosed, who presented with gradual regression of motor, speech, and cognition. MRI findings showed only mild periventriculat hyperintensities and finally were diagnosed by a genetic study.

Children with SSPE presented with primary measles infection followed by several asymptomatic years (6-15 on average), and then gradual, progressive psychoneurological deterioration, consisting of personality change, seizures, myoclonus, ataxia, photosensitivity, ocular abnormalities, spasticity, and coma.

Laboratory findings were disease specific. Anti-measles antibody was found in all diagnosed SSPE patients $(22.7 \%)$ cases, high $\alpha$-feto protein and abnormally low $\lg \mathrm{A}, \operatorname{lgG}$ in patients with ataxia telangiectasia $(11.76 \%)$, Low serum ceruloplasmin in Wilson disease (26.47\%), High ACTH (4.41\%) cases and low RBS (7.35\%) in ALD cases, Reduced $\mathrm{NCV}$ in $10.3 \%$ cases and specific genetic mutation was found in one patient with MLD\&one with Aicardi Goutiers syndrome.

Though all pediatric neurodegenerative disorders do not present with structural abnormalities at MRI, neuroimaging remains valuable support [7], [8]. Neuroimaging changes were found abnormal in $80.88 \%$ of NDDs of our studied patients. Among them, white matter hyperintensity in $29.41 \%$, Cerabeller atrophy in $13.25 \%$, Complete white matter hyperintensity with CSF density (VWMS) found in $2.94 \%$ cases of NDD, and Normal neuroimaging findings were found in $19.12 \%$ of patients. Here is a simplified flow diagram-based approach to leukodystrophies [5].

In one study with 213 patients, in leukodystrophic disorders including MLD, Krabbe disease, Canavan, PMD, PMD-like; $7.4 \%$ of patients had normal imaging; $3.7 \%$ had brain atrophy; $81.5 \%$ had white matter involvement, $3.7 \%$ had basal ganglia involvement [9].

Ophthalmic findings are common features of neurodegenerative disorders and, in addition are clinically important. In some patients, ophthalmic manifestations are the earliest symptoms of inherited neurodegenerative disease. Other than K F ring and sunflower cataract, optic atrophy, visual field defects, colour vision defects, ophthalmoplegia, retinal degeneration, pigmentary retinopathy, vision loss, telangiectatic vessels may be the common findings [10].

In this study, eye changes were found about $69.12 \%$ of patients with NDD. Among them, KF rings were found in $25.00 \%$, optic atrophy in $29.41 \%$, Telengiectasia in $11.76 \%$, normal eye evaluation found in $30.88 \%$ of cases.

So, the combination of MRI, careful clinical evaluation, and finally genetic evaluation especially next generation genetic sequencing holds promise for both expediting the diagnostic process and dramatically reducing the number of unresolved cases [11].

\section{CONCLUSION}

In our study, most common neurodegenerative disorders were leukodystrophy and wilson disease. Motor skill regression and speech regression, gait disorders were the common presenting features. Neuroimaging changes were found in more than three-fourth of cases of NDD. Commonest neuroimaging findings were white matter and basal ganglia hyper-intense signal changes in our study.

\section{RECOMMENDATION}

Neuroimaging may be very important tools for diagnosis of neurodegenerative disorders in children.

\section{AUTHOR CONTRIBUTIONS}

Prof. Dr. Gopen Kumar Kundu conceived and designed the study, collected data, and analyzed the data. Dr Rumana Islam played a role in clinical help. Dr. Noor- E-Sabah and Dr. ABM Mukib reviewed the article.

\section{ACKNOWLEDGMENT}

I acknowledge my teachers and resident of my department. 


\section{CONFLICT OF INTEREST}

\section{There was no conflict of interest.}

\section{REFERENCES}

[1] Stellitano LA, Winstone AM, Van der Knaap MS, Verity CM. Leukodystrophies and genetic leukoencephalopathies in childhood: a national epidemiological study. Developmental Medicine \& Child Neurology. 2016; 58(7): 680-689.

[2] Radke J, Stenzel W, Goebel HH. Neurometabolic and neurodegenerative diseases in children. Handbook of Clinical Neurology. 2018; 145: 133-146.

[3] Shubhankar M, Ajit PM. Approach to neurodegenerative disease in children: a short review. Progressing Aspects in Pediatrics and Neonatology. 2018;1(5)-77-82.

[4] Dyken P. and Krawiecki N. Neurodegenerative diseases of infancy and childhood. Annals of Neurology: Official Journal of the American Neurological Association and the Child Neurology Society. 1983; 13(4): 351-364.

[5] Gulati S, Jain P, Chakrabarty B, Kumar A, Gupta N, Kabra M. The spectrum of leukodystrophies in children: experience at a tertiary care centre from North India. Annals of Indian Academy of Neurology. 2016;19(3): 332.

[6] Kersten HM, Roxburgh RH, Danesh-Meyer HV. Ophthalmic manifestations of inherited neurodegenerative disorders. Nature Reviews Neurology. 2014;10(6): 349-362.

[7] Jan MM. Clinical approach to children with suspected neurodegenerative disorders. Neurosciences Journal. 2002; 7(1): 2-6.

[8] Schiffmann R, van der Knaap MS. Invited article: an MRI-based approach to the diagnosis of white matter disorders. Neurology. 2009; 72(8): 750-759.

[9] Verity C, Preece M. Surveillance for rare disorders by the BPSU. Archives of Disease in Childhood. 2002; 87(4): 269-271.

[10] Parikh S, Bernard G, Leventer RJ, van der Knaap MS, van Hove J, Pizzino A, et al. A clinical approach to the diagnosis of patients with leukodystrophies and genetic leukoencephelopathies. Molecular Genetics and Metabolism. 2015; 114(4): 501-515.

[11] Karimzadeh P, Jafari N, Biglari HN, Jabbehdari S, Zadeh SK, Abadi FA, et al. Neurometabolic diagnosis in children who referred as neurodevelopmental delay (a practical criterion, in Iranian pediatric patients). Iranian Journal of Child Neurology. 2016; 10(3): 73. 